\title{
DEUTERIUM ABUNDANCE IN THE LOCAL INTERSTELLAR MEDIUM
}

$$
\text { by Ferlet }{ }^{1}, R_{.}, \text {Gry, } C^{2} \text {, and Vidal-Madjar, } A^{1} \text {. }
$$

1. Institut d'Astrophysique de Paris

98bis Bd Arago - 75014 - Paris - France

2. IUE Observatory

Villafranca de1 Castillo - Appartado 54065 - Madrid - Spain

\section{ABSTRACT}

We underline the present situation of deuterium abundance evaluation in interstellar space and show that it should be $<10^{-5}$. Studiing in more detail the $\lambda$ Sco line of sight and having observed two NaI interstellar components toward that star, we $c$ an show that the $\mathrm{D} / \mathrm{H}$ evaluation made toward $\lambda \mathrm{Sco}$ is in fact related to the local interstellar medium (less than $10 \mathrm{pc}$ from the sun). Because this evaluation is also $<10^{-5}$ it is in stricking contrast with the one made toward $\alpha$ Aur $\left(D / H>1.810^{-5}\right)$ confirming the fact that the deuterium abundance in the local interstellar medium varies by at least a factor of two over few parsecs.

I INTRODUCTION

The most likely source of formation of light elements is during the first few minutes of the Universe, according to the so-called generally accepted Big Bang theory (Wagoner, 1973). They represent thus extraordinary cosmological probes, the primordial deuterium abundance in particular being probably one of the best tracers of the present baryon density of the Universe. On the other side, deuterium is almost always destroyed in stars and therefore should be also a probe of the chemical galactic evolution.

In that frame, one should expect a constant deuterium abundance in the nearby interstellar medium and until recently it was accepted that the measurements were in good agreement with the standard Big Bang predictions (see e.g. Austin, 1980). However, the question has now arised about the real cosmological significance of the deuterium observations and of the validity of the many assumptions made either in the Big Bang model or in the chemical evolution calculations (Vidal-Madjar and Gry 1984).

In effect, Vidal-Madjar et al (1983) showed that in the case of $\varepsilon$ Per, the DI line is blended by an HI component present in the stellar wind, changing in column-density and moving away from the star at approximately 80 $\mathrm{km} \mathrm{s} \mathrm{s}^{-1}$. Although this study could not give a significant $\mathrm{D} / \mathrm{H}$ ratio, it clearly showed that a stellar wind phenomenon can lead to an apparent overestimation of the deuterium abundance and may have influenced the previous $\mathrm{D} / \mathrm{H}$ evaluations.

To check this possibility Gry, Laurent and Vidal-Madjar (1983) carefully re-analysed the $\varepsilon$ Per Copernicus deuterium spectra and they pointed out that definite time-variations were present. More recently, Gry, 
Lamers and Vidal-Madjar (1984) have found similar variations in the Copernicus data toward three stars whose $\mathrm{D} / \mathrm{H}$ have been measured by York and Rogerson (1976) through the curve of growth technique : $\gamma^{2}$ vel, $\alpha$ Cru and $\alpha$ Vir. Therefore, one may suspect that the interstellar $\mathrm{D} / \mathrm{H}$ ratio toward these stars could be lower than previously evaluated (though probably still within the originally quoted errors) and thus more in agreement with the low $\mathrm{D} / \mathrm{H}$ values found toward the three Orion stars studied by Laurent, Vidal-Madjar and York (1979). Note that these Orion values already represented a strong constraint since they correspond to an average over three components detected on these sight-lines analysed with the profile fitting method. Note also that it is still possible to invoke a particularly strong astration toward the orion region.

As a preliminary conclusion and in view of the possible stellar contamination of the interstellar DI absorption line, we suggest that the $\mathrm{D} / \mathrm{H}$ interstellar ratio (within $1 \mathrm{kpc}$ of the Sun) as measured toward early type stars with the profile fitting analysis should be more likely of the order or less than $10^{-5}$, whereas the average of currently published values for hot stars is $1.45 \times 10^{-5}$.

\section{The $\lambda$ Sco line of sight}

Recently, York (1983) analysed the nearby B 1.5 IV star $\lambda$ Sco, also through the use of the profile fitting method in many lines of different species observed with Copernicus. He has detected five components in that line of sight. The main one (2) seems to be essentially neutral, while all the others are partly or strongly ionised. The $\mathrm{D} / \mathrm{H}$ evaluation was possible witp precision only in this component and led to the range $6 \times 10^{-6}<\mathrm{D} / \mathrm{H}<$ $10^{-5}$ deduced from the quoted $H$ and $D$ column densities and error bars. This result per-se confirms our previous conclusion and seems to show that in tpe nearby interstellar medium the $\mathrm{D} / \mathrm{H}$ abundance is in fact smaller than $10^{-5}$.

Since $\lambda$ Sco is only at about 120 pc from the Sun, one could question the fact that this evaluation is not only representative of the nearby ISM $(\sim 100 \mathrm{pc})$ but even of the local ISM ( $10 \mathrm{pc}$; LISM).

It could be therefore interesting to show that this main component observed toward $\lambda \mathrm{Sco}$ is in effect related to the immediate viccinity of the Solar system. Several studies have shown (see e.g. Vidal-Madjar et al, 1978 ; Mc Clintock et al, 1978 ; Bruhweiler and Kondo, 1982 ; Crutcher, 1982) that the LISM seems to contain a small neutral cloud in the general direction of the Sco-Cen association. Following the most recent compilation of observational information, Frisch and York (1983) showed that in the general direction of $\lambda$ Sco one should expect a neutral hydrogen column density $\mathrm{N}(\mathrm{HI}) \sim 5.10^{18} \mathrm{~cm}^{-2}$ located over the first $10 \mathrm{pc}$ away from the sun. This value is only a factor of 3 away from the total HI column density observed toward $\lambda$ Sco (N(HI) $1.710^{19} \mathrm{~cm}^{-2}$ ), $90 \%$ of which being locked in component 2. The HI cloud observed toward $\lambda$ Sco thus must be related to the local interstellar cloud (LIC). York (1983) gave evidences that thic HI component is probably quite compact and should spread over less than 5 pc. This is in very good agreement with the infered size of the LIC.

To be further convinced of the component 2 identification with the LIC, one should check two other physical parameters observed in this component : 
temperature and velocity. From the b-values evaluated in absorption lines corresponding to elements presenting very different masses, York (1983) has derived $b(H I)>b(D I)>b(O I)>b(A r I)$, concluding that component 2 cannot be cold and that the observed b-values are compatible with a medium at $T \simeq$ $10^{4} \mathrm{~K}$. This result is in very good agreement with other studies and in particular with the observed temperature of the interstellar medium flowing inside interplanetary space (see e.g. Bertaux, 1984).

The component 2 velocity also could be compared to the LISM velocities as predicted and studied by Crutcher (1982). Unfortunately, the Copernicus velocity information are not precise enough to clearly identify through velocity component 2 with the LISM since furthermore York (1983) found five components separated by about $10 \mathrm{~km} / \mathrm{s}$, a value in the range of the maximum absolute velocity error to be expected with the Copernicus instrument.

Since no optical observations of the quality required to obviously detect faint interstellar absorption lines and to have a precise velocity scale were available, we decided to reobserve the D-lines of the inferstellar neutral sodium toward $\lambda$ Sco with a spectral resolution of $3 \mathrm{~km}$ $s^{-1}$ and a signal to noise ratio high enough to be able to clearly detect equivalent width of the order of $1 \mathrm{~m} \AA$. Performed at the ESO - La Silla Observatory in Chile with a Reticon detector, the details of the instrumentation, observational procedure and data reduction can be found in Ferlet and Dennefeld (1984). For the present purpose, let just recall that the wavelength calibration is based on more than 13 thorium-argon lines emitted by a hollow-cathode lamp over the $50 \AA$ of the spectrum length giving an internal accuracy of $0.002 \AA$ or $0.1 \mathrm{~km} \mathrm{~s}^{-1}$. By comparing spectra taken at different epochs to be able to separate interstellar absorptions from telluric water vapor ones very numerous in the $\mathrm{NaI} D$ region, we have undoubtedly detected two interstellar components at $V_{0}=-26 \mathrm{~km} \mathrm{~s}^{-1}$ and at $V_{0}=-17 \mathrm{~km} \mathrm{~s}^{-1}$ (see Figure 1).

From the study of York (1983) it is clear that one should expect some $\mathrm{NaI}$ counterpart (although weak) only from component 2 (the main $\mathrm{H}_{2}$ component) and component 3 , a component in which there is in fact a similar total hydrogen content as in component 2, but in which hydrogen is at about $90 \%$ ionised. Nevertheless this component is still weakly ionised and $\mathrm{NaI}$ could be significantly present in it. All other components are much too weak or too ionized to show any $\mathrm{NaI}$.

Component 2 and 3 have a velocity separation of $9 \mathrm{~km} \mathrm{~s}^{-1}$ in remarkable agreement with the observed velocity separation of the two NaI components which is also observed to be of $9 \mathrm{~km} \mathrm{~s}^{-1}$.

From Crutcher (1982) analysis, one should observe in the direction of the star $\lambda$ Sco an heliocentric velocity for the LISM of $-23 \mathrm{~km} \mathrm{~s}$. This result thus shows independently that component 2 is the more likely to be related to the LISM since as indicated by Crutcher (1982) most of the interstellar components observed toward nearby stars seem to fall within \pm 3 $\mathrm{km} \mathrm{s}^{-1}$ from the predicted values.

In conclusion, from that study, we have showed that all parameters related to component 2 observed by York (1983) toward $\lambda$ Sco are in excellent agreement with the LISM parameters (column density, velocity, temperature) and thus seem to be clearly related to the LIC located at the edge of the solar system. 


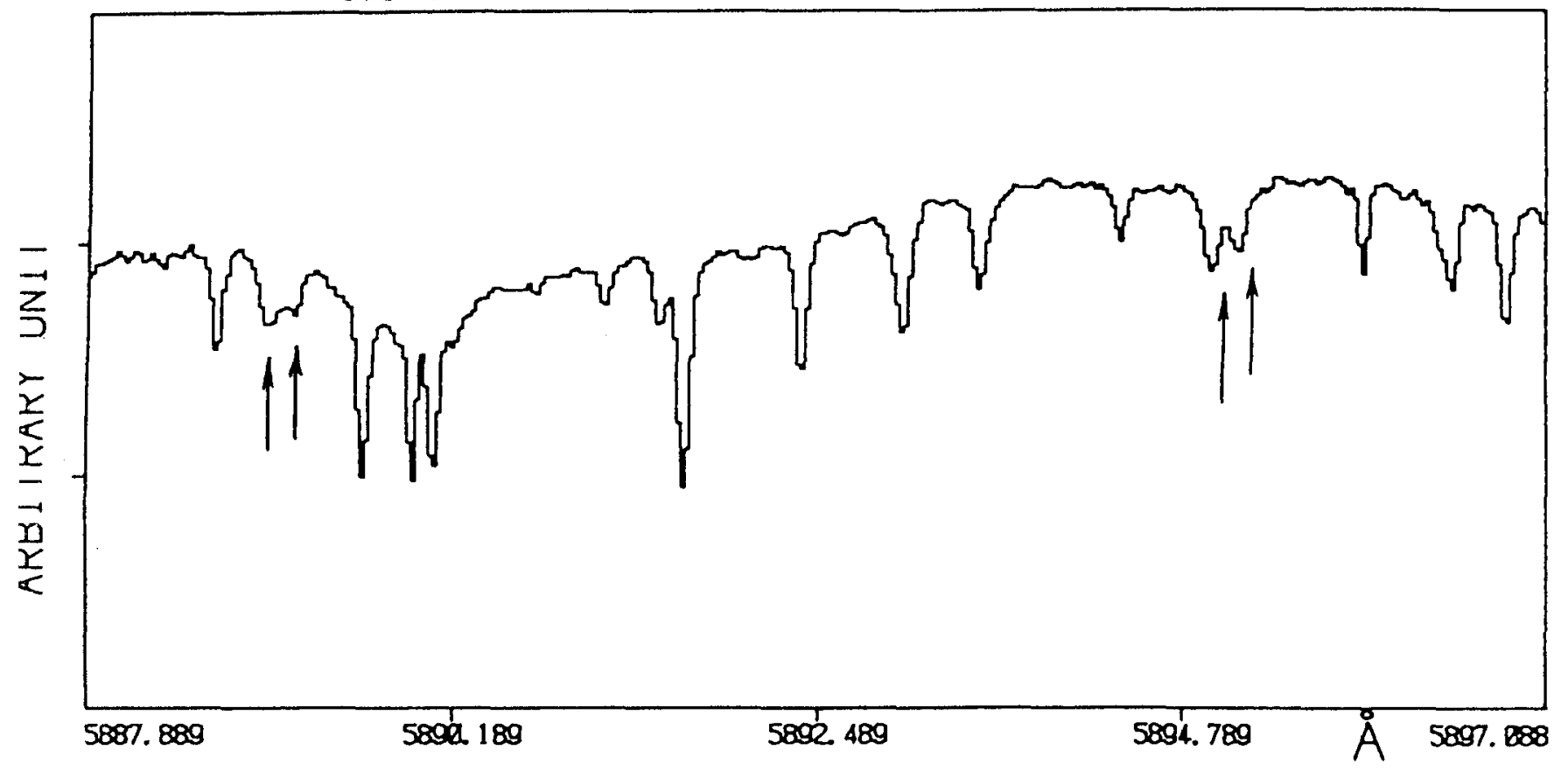

Figure 1: Reticon spectrum of the interstellar D-lines of neutral sodium toward $\lambda$ Sco, after corrections for the read-out noise and pixel to pixel sensitivity. The positions of the detected $\mathrm{NaI}$ absorptions are indicated. All other lines are telluric water vapor absorptions, superposed on broad stellar lines. The two interstellar lines near 5889 \& are almost uncontaminated by water vapor lines while the two corresponding ones near $5895 \AA$ are heavily blended with two telluric lines.

\section{Discussion and Conclusion}

Coming back to the deuterium evaluation, we now see that the $\lambda$ Sco evaluation is not only related to the nearby interstellar medium but also to the LISM. It could be thus interesting to compare this value with the previous estimations of $\mathrm{D} / \mathrm{H}$ in the LISM.

These estimations are very difficult due to the unknown stellar Lyman $\alpha$ prof $i$ le of the target cool stars. Nevertheless, in the direction of $\alpha$ Aur, the Copernicus data are of very high quality and the conclusion of two independant groups (Dupree, Baliunas and Shipman, 1977 ; and Mc Clintock et al, 1978) are very similar, showing that at least in that direction the $D / H$ value should be larger than $1.810^{-5}$, a more probable value beeing around $2.510^{-5}$. This result is in stricking disagreement with the $\lambda$ Sco one, showing that the $\mathrm{D} / \mathrm{H}$ ratio in the LISM varies by at least a factor of 2 (and more probably by a factor of 4 ) over a few parsecs.

Although this peculiar behaviour of the deuterium abundance in the LISM is clearly shown in at least two lines of sight, it is still difficult to 
try to explain it by precise mechanisms due to lack of observational material (see some possibilities suggested by Vidal-Madjar et al, 1978). To improve the situation, more lines of sight toward nearby stars observed with Copernicus should be analysed with the profile fitting approach in order to give the component by component characteristics of the line of sight. Certainly also, one will have to wait for ST - HRS data which should improve considerably the situation.

\section{References:}

Anderson, R.C., Henry, R.C., Moos, H.W., and Linsky, J.L., 1978, Ap.J., 226, 883

Anderson, R.C., and Weiler, E.J., 1978, Ap.J., 224, 143

Austin, S.M., 1980, Prog. in Part. and NuCl. Phys.

Baliunas, S.L., and Dupree, A.K., 1979, Ap.J., 227, 870

Bertaux, J.L., 1984, these proceedings.

Bruhweiler, F.C. and Kondo, Y., 1982, Ap.J., 259, 232

Crutcher, R.M., 1982, Ap.J., 254, 82

Dupree, A.K., Baliunas, S.L., and Shipman, H.L., Ap.J., 218, 361

Ferlet, R. and Dennefeld, M., 1984, Astron. Astrophys., in press

Frisch, P.C., and York, D.G., 1983, Ap.J. Letters, 271, L59.

Gry, C., Lamers, H.J.G.L.M., and Vidal-Madjar, A., 1984, Astron. Astrophys., in press

Gry, C., Laurent, C. and Vidal-Madjar, A., 1983, Astron. Astrophys., 124, 99 Laurent, C., Vidal-Madjar, A. and York, D.G., 1979, Ap.J., 229, 923

Mc Clintock, W., Henry, R.C., Linsky, J.L., and Moos, H.W., 1978, Ap.J., 225,465

Stokes, G.M., 1978, Ap.J. Suppl., 36, 115

Vidal-Madjar, A. and Gry, C., 1984, Astron. Astrophys., in press

Vidal-Madjar, A., Laurent, C., Bruston, P., and Audouze, J., 1978, Ap.J., 223, 589

VidaT-Madjar, A., Laurent, C., Gry, C., Bruston, P., Ferlet, R. and York, D.G., 1983, Astron. Astrophys., 120, 58

Wagoner, R.V., 1973, Ap.J., 179, 343

York, D.G., 1983, Ap.J., 264, 172

York, D.G. and Rogerson, J.B., 1976, Ap.J., 203, 378 